\title{
Bicuspid stenotic aortic valves: clinical characteristics and morphological assessment using MRI and echocardiography
}

\author{
I. C. Joziasse - A. Vink • M. J. Cramer • M. F. M. van Oosterhout • \\ L. A. van Herwerden - R. Heijmen - G. T. J. Sieswerda • B. J. M. Mulder • \\ P. A. Doevendans
}

Published online: 8 February 2011

(C) The Author(s) 2011. This article is published with open access at Springerlink.com

\begin{abstract}
Background Bicuspid aortic valve (BAV) is one of the most common congenital heart defects with a population prevalence of $0.5 \%$ to $1.3 \%$. Identifying patients with $\mathrm{BAV}$ is clinically relevant because BAV is associated with aortic stenosis, endocarditis and ascending aorta pathology.
\end{abstract}

\section{C. Joziasse ( $\square)$ \\ Department of Cardiology, University Medical Center Utrecht, Housepost Q 05.2.314, PO Box 85500, 3508 GA Utrecht, the Netherlands \\ e-mail: I.C.Joziasse@umcutrecht.nl}

M. J. Cramer · G. T. J. Sieswerda • P. A. Doevendans Department of Cardiology, University Medical Center Utrecht, GA Utrecht,

the Netherlands

\section{A. Vink}

Department of Pathology, University Medical Center Utrecht,

GA Utrecht, the Netherlands

M. F. M. van Oosterhout

Department of Pathology, St. Antonius Hospital Nieuwegein, EM Nieuwegein, the Netherlands

\section{A. van Herwerden}

Department of Cardiothoracic surgery,

University Medical Center Utrecht,

GA Utrecht, the Netherlands

\section{R. Heijmen}

Department of Cardiothoracic Surgery,

St Antonius Hospital Nieuwegein,

EM Nieuwegein, the Netherlands

\section{B. J. M. Mulder}

Department of Cardiology, Academic Medical Center Amsterdam,

DD Amsterdam, the Netherlands
Methods and Results Patients with severe aortic stenosis necessitating aortic valve replacement surgery were included in this study. All dissected aortic valves were stored in the biobank of the University Medical Centre Utrecht. Additionally to the morphological assessment of the aortic valve by the surgeon and pathologist, echocardiographic and magnetic resonance imaging (MRI) images were evaluated. A total of 80 patients were included of whom 32 (40\%) were diagnosed with BAV by the surgeon (gold standard). Patients with BAV were significantly younger (55 vs 71 years) and were more frequently male. Notably, a significant difference was found between the surgeon and pathologist in determining valve morphology. MRI was performed in 33\% of patients. MRI could assess valve morphology in $96 \%$ vs $73 \%$ with echocardiography. The sensitivity of MRI for BAV in a population of patients with severe aortic stenosis was higher than echocardiography $(75 \%$ vs $55 \%$ ), whereas specificity was better with the latter (91\% vs 79\%). Typically, the ascending aorta was larger in patients with BAV.

Conclusion Among unselected patients with severe aortic valve stenosis, a high percentage of patients with BAV were found. Imaging and assessment of the aortic valve morphology when stenotic is challenging.

Keywords Bicuspid aortic valve - Magnetic resonance imaging $\cdot$ Echocardiography $\cdot$ Morphology assessment

\section{Introduction}

Bicuspid aortic valve (BAV) is one of the most common congenital heart defects with a population prevalence of $0.5 \%$ to $1.3 \%[1-3]$. The defect is considered to be a 
heritable disorder, with a family recurrence rate of approximately $35 \%$ [4]. Recent studies show that mutations in the NOTCH1 gene are associated with BAV [5-7]. Bicuspid aortic valves progress more rapidly into regurgitation or stenosis of the valve [8]. This results in a higher occurrence of aortic valve replacement, especially at younger age [8]. Additionally, BAV are more susceptible than tricuspid aortic valves (TAV) to nest bacteria or other organisms, leading to endocarditis [9]. BAV is not only a peculiar valve morphology leading to specific valve pathology, it is also frequently associated with (asymptomatic) ascending aorta dilatation which leads to an increased susceptibility to ascending aortic aneurysms and aortic dissection [10-14]. Aortic elasticity measurements of BAV patients suggest that diminished aortic elasticity is at least part of its causation [15].

Unfortunately, BAV patients frequently remain undiagnosed until the manifestation of symptoms. Therefore, screening and detection of patients is warranted. In 2007, we started a study, designed for the detection of genetic mutations/variations in BAV patients in a cohort of patients accepted for aortic valve replacement in the University Medical Centre Utrecht and the St Antonius Hospital Nieuwegein. In this report, we describe the clinical characteristics of the included patients thus far. Additionally, the morphological assessment of the aortic valve by the pathologist and surgeon were compared. Sensitivity and specificity for the diagnosis of BAV by magnetic resonance imaging (MRI) and echocardiography in a population with severe aortic stenosis was determined as well.

\section{Material and Methods}

\section{Study Design}

This study was designed as a multicentre observational cross-sectional study. The participating centres were the University Medical Centre Utrecht and the St Antonius Hospital, Nieuwegein, the Netherlands. The study was reviewed and approved by the ethics boards of both hospitals. The methodology and privacy regulations are in accordance with Dutch and European privacy protection laws.

Study Population

All patients between 18 and 80 years of age who were scheduled for aortic valve replacement between July 2007 and 2009 were eligible for participation in this study. Patients with endocarditis and prior aortic valve replacement were excluded. As this study was primarily designed for the identification of genetic variation associated with BAV, patients with a genetic diagnosis such as Marfan's disease, Down syndrome, Turner syndrome and Noonan syndrome were excluded from participation as well.

\section{Collecting Data and Tissue Preparation}

Informed consent was obtained before surgery. During valve surgery, $10 \mathrm{ml}$ of blood was obtained for genomic DNA extraction. The aortic valve was photographed in vivo and excised. In case of a BAV, the surgeon documented the presence or absence of a raphe and its position on a standardised form according to the classification system of Sievers and Schmidtke [16]. After excision, the aortic valve leaflets were collected on ice by dedicated staff and immediately taken to the Pathology Department. Next, the aortic valve leaflets were inspected, measured and photographed by a cardiovascular pathologist (AV). Each of the aortic valve leaflets was subsequently bisected. Half of the valve leaflet was snap frozen in liquid nitrogen and stored at $-80^{\circ} \mathrm{C}$ for further analyses in the UMC Utrecht biobank. The remaining half was fixed in formalin, decalcified in EDTA and embedded in paraffin. Haematoxylin and eosin as well as Elastica van Giesson stainings were performed on three micron slides for routine histopathological purposes.

\section{Echocardiography and MRI}

An MRI investigation of the aorta and aortic valves was made for identification of either a BAV or TAV and for identification of aortic root dilatation and coarctation of the aorta before surgery. First, the aorta was examined in the transverse imaging planes. Then, imaging planes perpendicular to the long axis were used to assess the diameters of the aortic root, sinotubular junction (STBJ), ascending aorta, aortic arch and the descending aorta accurately. The morphology of the aortic valve was evaluated in specific imaging planes perpendicularly or longitudinally oriented through the aortic valve. All images were evaluated by a cardiologist experienced in assessing cardiac MRIs. Echocardiograms from the referring hospitals were reviewed by at least two investigators (ICJ, MJC). The aortic valve was examined in the left parasternal long-axis view (a bicuspid aortic valve shows a doming configuration when it opens during systole). M-mode was used to determine 
whether the valve closed centrally and the short-axis view was used for determining further valve morphology ('fish-mouth' opening in true bicuspid aortic valve, or somewhat altered with a fused commissure). If the aortic valve was too calcified to determine the valve morphology, then the valve was defined 'undetermined'. The aorta was measured at several positions in the parasternal long-axis view. Measurements were made at the level of the annulus, sinus valsalvae (aortic root) STBJ and the ascending aorta, measured $4 \mathrm{~cm}$ from the annulus in diastole. The aortic root was also evaluated at the M-mode recording.

\section{Data Analysis}

Patients were divided into two groups based on the morphological assessment of the valves at surgery: a BAV and TAV group. For comparing clinical characteristics, an unpaired $t$ test was used for continuous variables and a $\chi^{2}$ test for comparing categorical variables. For the comparison of aortic diameter means between the bicuspid and tricuspid groups, analysis of covariance was used with as covariates aortic valve morphology, hypertension and age. A paired $t$ test was used to compare differences in echocardiographic evaluation of aortic size versus aortic diameters measured with MRI. The McNemar test was used for determining differences between surgical and pathological evaluation of aortic valve morphology.

Sensitivity and specificity were calculated with the Clinical Calculator 1 (http://faculty.vassar.edu/lowry/clin1. html). Two-sided $p$ values below 0.05 were considered statistically significant. SPSS for Windows, (release 15.0.0. 2006, Chicago SPSS Inc.) was used for all other statistical analyses.

Table 1 Clinical characteristics

$A V A$ aortic valve area, $N S$

nonsignificant

${ }^{\mathrm{a}}$ Unadjusted $p$ values

${ }^{\mathrm{b}}$ When adjusted for age, hypertension is not significantly associated with a tricuspid aortic valve

\section{Results}

\section{Clinical Characteristics}

A total of 80 patients were included. Clinical characteristics are shown in Table 1 . Of the patients, $65 \%$ were male and mean age at operation was 68.5 years; $51 \%$ of the patients were hypertensive and $25 \%$ were obese. Diabetes and hypercholesterolaemia occurred in $10 \%$ and $31 \%$ of all patients, respectively. BAV was more often observed in males than in females $(p=0.004$; Table 1). Patients with BAV where younger than patients with a TAV (55 vs. 71 years, $p<$ 0.0001; Table 1). BAV patients had less hypertension and overweight as compared with TAV patients, although the number of patients with these risk factors was limited. When correcting for the confounding factor age, hypertension was no longer associated with a tricuspid aortic valve. None of the other known risk factors associated with typical cardiovascular disease were significantly different between the two groups.

\section{Assessment of Aortic Valve Morphology}

\section{Surgeon Versus Pathologist}

A significant difference between the surgeon and pathologist in determining valve morphology was found (Table 2). Especially when the surgeon diagnosed a TAV, the pathologist more often assessed the aortic valve to be bicuspid with a raphe (type 1 according to the classification system of Sievers and Schmidtke; Fig. 1) [16]. For further analysis, the surgeon's assessment of valve morphology was used as the gold standard. In total, $38 \%$ of patients were found to have $\mathrm{BAV}, 57 \%$ had a TAV and in the remaining $5 \%$ the valve morphology could not be determined.

\begin{tabular}{lcccl}
\hline & Overall $(n=80)$ & Tricuspid $(n=48)$ & Bicuspid $(n=32)$ & $p$ value $^{\mathrm{a}}$ \\
\hline Male (\%) & $54(65.1)$ & $24(51)$ & $26(84)$ & 0.004 \\
Age at surgery & $68.5(56.5-74)$ & $71(68-75)$ & $55(48-66)$ & $<0.0001$ \\
AVA $\left(\mathrm{cm}^{2}\right)$ & $0.76(0.23)$ & $0.78(0.23)$ & $0.72(0.22)$ & $\mathrm{NS}$ \\
Peak gradient & $78(22)$ & $78(21)$ & $75(13)$ & $\mathrm{NS}$ \\
Hypertension (\%) & $41(51)$ & $31(65)$ & $10(31)$ & $0.003^{\mathrm{b}}$ \\
Diabetes (\%) & $8(10)$ & $7(15)$ & $1(3)$ & $\mathrm{NS}$ \\
Obese (\%) & $19(24)$ & $16(33)$ & $3(5)$ & 0.010 \\
Hypercholesterolaemia (\%) & $28(31)$ & $20(42)$ & $8(25)$ & $\mathrm{NS}$ \\
Smoking (\%) & & & & \\
Never & $37(46)$ & $23(48)$ & $8(44)$ & \\
Past & $15(19)$ & $7(16)$ & $6(19)$ & \\
Recently stopped & $13(16)$ & $11(23)$ & $4(13)$ & $\mathrm{NS}$ \\
Current smoker & $15(19)$ & & \\
\hline
\end{tabular}


Table 2 Aortic valve morphology; surgery compared with pathologist, ECHO and MRI

\begin{tabular}{llccr}
\hline & & \multicolumn{3}{c}{ Surgical assessment } \\
\cline { 3 - 5 } & & Bicuspid & Tricuspid & Total \\
\hline Pathologist & Bicuspid & 29 & 14 & 43 \\
& Tricuspid & 2 & 35 & 37 \\
\multirow{4}{*}{ Echocardiography } & Total & 31 & 49 & 80 \\
& Bicuspid & 6 & 2 & 8 \\
& Tricuspid & 5 & 21 & 26 \\
MRI & Total & 11 & 23 & 34 \\
& Bicuspid & 9 & 3 & 12 \\
& Tricuspid & 3 & 11 & 14 \\
& Total & 14 & 12 & 26 \\
\hline
\end{tabular}

\section{MRI and ECHO}

Diagnostic accuracy of MRI (Fig. 2) was 79\%. In addition, MRI was able to correctly identify a bicuspid aortic valve in $75 \%$ (43-93) of cases (sensitivity). Specificity of MRI was 0.79 (0.49-0.94; Table 2). In one ( 4\%) patient, aortic valve morphology could not be determined with MRI. The positive predictive value was $75 \%(43-93)$ and the negative predictive value was $79 \%$ (49-94).

In $27 \%$ of patients, the morphology of the aortic valve could not be determined by echocardiography due to severe calcifications of the aortic valves $(\sim 70 \%)$ or insufficient window quality $(\sim 30 \%)$. Of the remaining echocardiograms (Fig. 2), the diagnostic accuracy was $66 \%$. In this population of patients with severe aortic stenosis, sensitivity was only $0.54(0.25-0.82)$, which is somewhat smaller than MRI. Echocardiography was, on the other hand, more specific than MRI for excluding the presence of BAV $(0.91(0.25-0.82)$ vs 0.79 (0.49-0.94); Table 2). The positive and negative predictive values were $75 \%$ (36-96) and $80 \%(60-92)$, respectively.

The maximum aortic diameter was defined as the maximum aortic diameter measured at three to five measuring levels at ECHO and MRI, respectively. Measurements were compared between patients with a bicuspid and tricuspid aortic valve. The ascending aorta was significantly wider in the bicuspid compared with the tricuspid patients, even when corrected for possible confounders such as hypertension and age ( $p=0.021$; Table 3$)$. Additionally, the maximum diameter measured in any of the five measurement sites was significantly broader in the BAV group as
Fig. 1 Photographs of aortic valve during surgery and during inspection by the pathologist. a, b Patient with severe aortic stenosis and diagnosed with a type 0 bicuspid aortic valve by both surgeon (a) and pathologist (b); c, d patient with tricuspid aortic valve at surgery (c), this photograph shows a clear view of the presence of three separate valve leaflets. d Excised valves of the same patient. It is hard to identify three separate valve leaflets after excision and this aortic valve was determined to be a type 1 bicuspid aortic valve (with raphe) by the pathologist
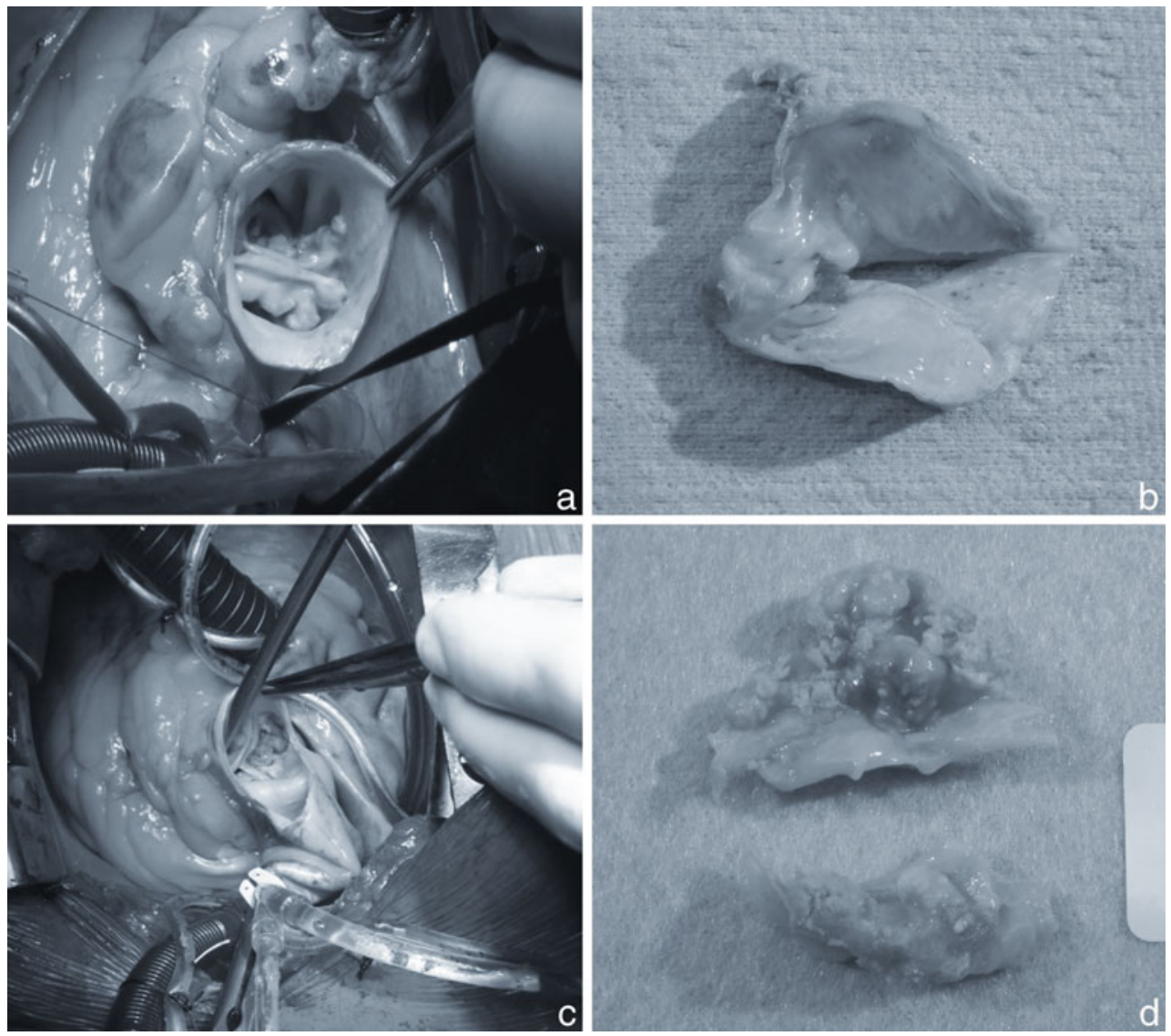
Fig. 2 a MRI of a patient with a tricuspid aortic valve. b Patient with a tricuspid aortic valve with echocardiography. Although, this echocardiogram was analysable, the view of the aortic valve is markedly inferior compared with MRI
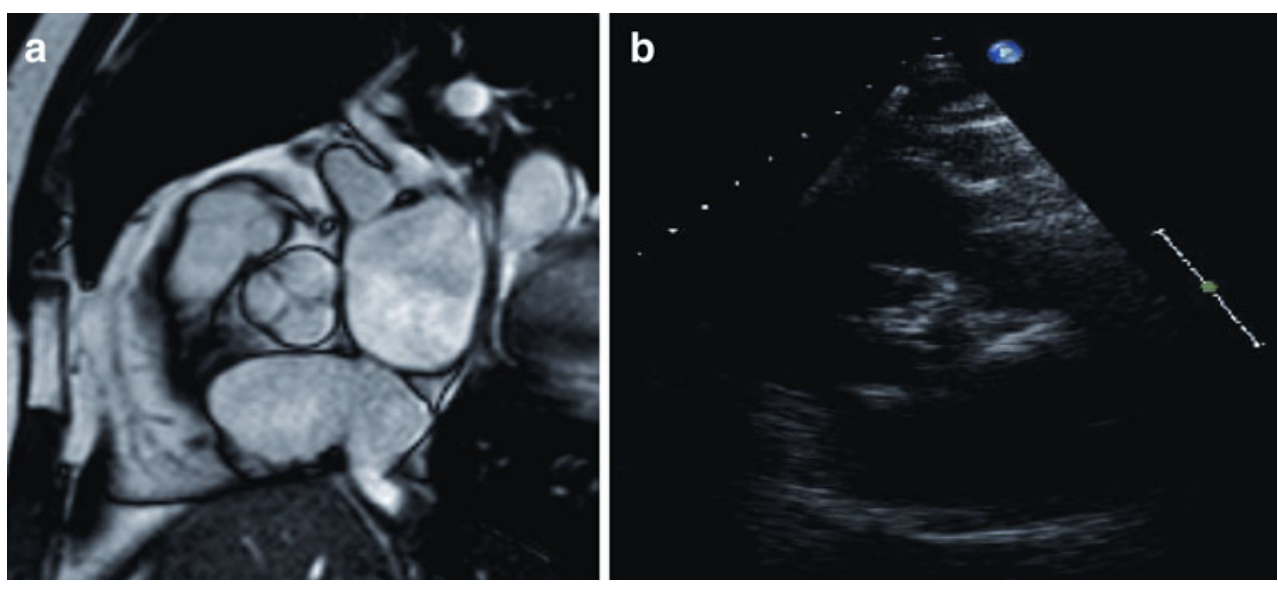

compared with the TAV group ( $p=0.001$; Table 3$)$. When assessing aortic dimensions with echocardiography similar results were found; the ascending aorta $(29.7 \mathrm{~mm}$ (TAV) vs $33.4 \mathrm{~mm}(\mathrm{BAV}), p=0.045)$ and maximum diameter ( 33.2 vs $37.8 \mathrm{~mm}, p=0.018$ ) were significantly associated with the presence of BAV. In addition, the aortic diameters of the sinus valsalvae and the STBJ demonstrated a trend towards a wider aorta in the BAV group ( $p=0.087$ and $p=0.067$, respectively), even when corrected for hypertension and age (Table 3 ). The aortic dimensions of the sinus valsalvae and STBJ were comparable between MRI and echocardiography. The ascending aortic dimensions, on the other hand, were systematically underestimated with echocardiography (mean difference $3.5 \mathrm{~mm}(0.574-6.49 \mathrm{~mm}, p=0.023)$ ).

\section{Discussion}

To the best of our knowledge, this is the first study in which valve morphology was assessed systematically by the surgeon, pathologist, MRI and echocardiography in an unselected population of patients with severely stenotic aortic valves. We observed a significant difference in the assessment of aortic valve morphology between the surgeon and pathologist. A possible explanation for the discrepancy between the assessment of the surgeon and pathologist is the fact that the surgeon can judge the valve in the anatomical position, whereas the pathologist has to classify a valve after dissection. It is therefore difficult for the pathologist to determine whether the valve leaflets are congenitally fused (raphe) or fused due to the degenerative disease itself. Especially in calcified valves that are sometimes dissected in multiple fragments, classification by the pathologist is less reliable. Although tissue examination of the pathologist is considered the gold standard in most diseases, this is not the case in the classification of bicuspid aortic valves.

\section{Clinical Characteristics}

In this report, $40 \%$ of patients with severe aortic valve stenosis were found to have a $\mathrm{BAV}$, which is comparable with previous reported frequencies [8]. Hypertension was found in $51 \%$ of all patients who participated in our study, which is higher than expected considering population prevalence of hypertension in the Netherlands $(27 \%$ to $34 \%$ for persons $>60$ years of age) $[17,18]$. This difference could be merely due to small numbers, but other reports have also described the increased prevalence of hypertension in patients with aortic stenosis versus a control population of individuals without relevant valve disease [19]. Therefore, this difference appears a real finding. These data therefore suggest that hypertension is associated with aortic stenosis.
Table 3 Aortic dimensions measured with MRI and ECHO

All diameters are in $\mathrm{mm}$ NS nonsignificant, STBJ sinotubular junction, $T A$ thoracic aorta

${ }^{a} p$ values are corrected for hypertension and age

\begin{tabular}{llllllll}
\hline Aortic position & \multicolumn{2}{l}{ MRI (mean (SD)) } & & & ECHO (mean (SD)) & \\
\cline { 2 - 3 } \cline { 7 - 8 } & Tricuspid & Bicuspid & $p$ value $^{*}$ & & Tricuspid & Bicuspid & $p$ value $^{\mathrm{a}}$ \\
\hline Sinus valsalva & $32.9(4.38)$ & $35.3(7.18)$ & NS & & $32.8(4.20)$ & $36.4(5.11)$ & NS \\
STBJ & $29.7(3.55)$ & $29.3(3.47)$ & NS & & $27.8(4.11)$ & $32.1(5.43)$ & NS \\
Ascending aorta & $33.8(5.18)$ & $39.5(7.01)$ & 0.021 & & $29.7(5.24)$ & $33.4(5.42)$ & 0.045 \\
Aortic arch & $27.0(3.12)$ & $27.9(4.85)$ & NS & & & \\
Descending TA & $25.7(0.70)$ & $25.5(0.75)$ & NS & & & \\
Max diameter & $35.7(4.36)$ & $40.5(6.40)$ & 0.001 & & $33.2(4.09)$ & $37.8(4.80)$ & 0.018 \\
\hline
\end{tabular}


Surprisingly, overweight was significantly associated with a tricuspid aortic valve even after adjustment for age. A high fat/high carbohydrate diet induces aortic valve disease in mice, independently of cholesterol level [20]. Therefore, overweight is likely to be a risk factor for the development of aortic stenosis. Bicuspid aortic valves are already more susceptible for aortic valve stenosis than tricuspid aortic valves. Therefore, additional risk factors presumably more explain aortic valve stenosis in tricuspid than bicuspid aortic valves. Certainly, as numbers were small, this finding can also be due to chance.

Additionally, significantly more male than female patients were observed with BAV ( $84 \%$ vs $51 \%)$. Similar gender differences in the incidence and prevalence of BAV have been previously reported [1,21-23].

\section{Imaging of the Aortic Valve and Aortic Dimensions}

BAV is associated with aortic dilatation which leads to an increased susceptibility to aortic dissection and ascending aortic aneurysms [10-14]. Therefore, it is important to recognise aortic valve morphology for future follow-up (e. g. regular assessment of the ascending aorta) and possibly intervention. Furthermore, for risk stratification in family members, knowledge of valve morphology can be important as well. Imaging of the aortic valve when severely stenotic is challenging. Due to the severity of stenosis and calcified nature of the aortic valves, echocardiograms were frequently unable to differentiate between TAV and BAV. As predicted, MRI was able to assess aortic valve morphology more frequently than echocardiography $(96 \%$ vs. $73 \%$ ). MRI appeared more sensitive for detecting of BAV in this population than echocardiography ( 0.75 vs 0.55). Whilst MRI was more sensitive, echocardiography on the other hand appeared more specific (0.91 vs 0.79$)$ in this patient group. Certainly, patients who present to the cardiologist in a less advanced stage may presumably be better identified with either imaging techniques. From a cost-effectiveness perspective, echocardiography would still be the first choice. Namely, in those patients with adequate echocardiographic images, echocardiography has been reported to be both highly sensitive $(0.79-0.92)$ and specific $(0.93-0.96)[24,25]$. When the echocardiograms prove unanalysable or when in doubt, MRI can be useful to come to a diagnosis.

MRI is considered the gold standard for the determination of aortic dimensions. In this report aortic dimensions were assessed with both echocardiography and MRI. Both MRI and echocardiography demonstrated a significantly larger ascending aorta in BAV patients compared with tricuspid aortic valve patients. This pattern is comparable with previous reports of aortic diameters in BAV patients [11-13]. When comparing aortic dimensions assessed by means of MRI and ECHO, the ascending aortic dimensions were systematically underestimated with echocardiography. Therefore, when the ascending aorta appears large at echocardiography, it is important to consider evaluating the aorta with MRI as standardised care. The role of other advanced imaging techniques such as computerised tomography was not evaluated in this report.

\section{Conclusion}

Among unselected patients with severe aortic valve stenosis, a high percentage of patients with BAV were found. Patients with BAV were significantly younger and more frequently male. Typically, the ascending aorta was larger in patients with BAV. Notably, a significant difference between the surgeon and pathologist in determining valve morphology was found. Although tissue examination of the pathologist is considered the gold standard in most diseases, this is not the case in the classification of bicuspid aortic valves. Therefore in this study, the gold standard for assessing these stenotic valves was the appraisal of the surgeon. Imaging of the aortic valve with echocardiography appeared challenging due to the severe calcified aortic valves. MRI is more sensitive but less specific than echocardiography in the classification of aortic valves.

Open Access This article is distributed under the terms of the Creative Commons Attribution Noncommercial License which permits any noncommercial use, distribution, and reproduction in any medium, provided the original author(s) and source are credited.

\section{References}

1. Movahed MR, Hepner AD, Ahmadi-Kashani M. Echocardiographic prevalence of bicuspid aortic valve in the population. Heart Lung Circ. 2006;15:297-9.

2. Tutar E, Ekici F, Atalay S, et al. The prevalence of bicuspid aortic valve in newborns by echocardiographic screening. Am Heart J. 2005;150:513-5.

3. Hoffman JI, Kaplan S. The incidence of congenital heart disease. J Am Coll Cardiol. 2002;39:1890-900.

4. Huntington K, Hunter AG, Chan KL. A prospective study to assess the frequency of familial clustering of congenital bicuspid aortic valve. J Am Coll Cardiol. 1997;30:1809-12.

5. Garg V, Muth AN, Ransom JF, et al. Mutations in NOTCH1 cause aortic valve disease. Nature. 2005;437:270-4.

6. Mohamed SA, Aherrahrou Z, Liptau H, et al. Novel missense mutations (p.T596M and p.P1797H) in NOTCH1 in patients with bicuspid aortic valve. Biochem Biophys Res Commun. 2006;345:1460-5.

7. Martin LJ, Ramachandran V, Cripe LH, et al. Evidence in favor of linkage to human chromosomal regions $18 \mathrm{q}, 5 \mathrm{q}$ and $13 \mathrm{q}$ for bicuspid aortic valve and associated cardiovascular malformations. Hum Genet. 2007;121:275-84. 
8. Roberts WC, Ko JM. Frequency by decades of unicuspid, bicuspid, and tricuspid aortic valves in adults having isolated aortic valve replacement for aortic stenosis, with or without associated aortic regurgitation. Circulation. 2005;111:920-5.

9. Lamas CC, Eykyn SJ. Bicuspid aortic valve - a silent danger: analysis of 50 cases of infective endocarditis. Clin Infect Dis. 2000;30:336-41.

10. Larson EW, Edwards WD. Risk factors for aortic dissection: a necropsy study of 161 cases. Am J Cardiol. 1984;53:849-55.

11. Hahn RT, Roman MJ, Mogtader AH, et al. Association of aortic dilation with regurgitant, stenotic and functionally normal bicuspid aortic valves. J Am Coll Cardiol. 1992;19:283-8.

12. Keane MG, Wiegers SE, Plappert T, et al. Bicuspid aortic valves are associated with aortic dilatation out of proportion to coexistent valvular lesions. Circulation. 2000;102:III35-9.

13. Pachulski RT, Weinberg AL, Chan KL. Aortic aneurysm in patients with functionally normal or minimally stenotic bicuspid aortic valve. Am J Cardiol. 1991;67:781-2.

14. Roberts CS, Roberts WC. Dissection of the aorta associated with congenital malformation of the aortic valve. J Am Coll Cardiol. 1991;17:712-6.

15. Yap SC, Nemes A, Meijboom FJ, et al. Abnormal aortic elastic properties in adults with congenital valvular aortic stenosis. Int $\mathrm{J}$ Cardiol. 2008;128:336-41.

16. Sievers HH, Schmidtke C. A classification system for the bicuspid aortic valve from 304 surgical specimens. J Thorac Cardiovasc Surg. 2007;133:1226-33.

17. van den Berg PJ, van Dalsen CL, de Rooij RA, et al. Cardiovascular health check in the elderly in one general practice: does it offer new information and lead to interventions? Fam Pract. 1999;16:389-94.

18. Mennen LI, Witteman JC, Geleijnse JM, et al. Risk factors for cardiovascular diseases in the elderly; the ERGO study (Erasmus Rotterdam Health and the Elderly). Ned Tijdschr Geneeskd. 1995;139:1983-8.

19. Rossebo AB, Pedersen TR, Allen C, et al. Design and baseline characteristics of the simvastatin and ezetimibe in aortic stenosis (SEAS) study. Am J Cardiol. 2007;99:970-3.

20. Drolet MC, Roussel E, Deshaies Y, et al. A high fat/high carbohydrate diet induces aortic valve disease in C57BL/6 J mice. J Am Coll Cardiol. 2006;47:850-5.

21. Della CA, Bancone C, Quarto C, et al. Predictors of ascending aortic dilatation with bicuspid aortic valve: a wide spectrum of disease expression. Eur J Cardiothorac Surg. 2007;31:397-404.

22. Basso C, Boschello M, Perrone C, et al. An echocardiographic survey of primary school children for bicuspid aortic valve. Am J Cardiol. 2004;93:661-3.

23. Michelena HI, Desjardins VA, Avierinos JF, et al. Natural history of asymptomatic patients with normally functioning or minimally dysfunctional bicuspid aortic valve in the community. Circulation. 2008;117:2776-84.

24. Chan KL, Stinson WA, Veinot JP. Reliability of transthoracic echocardiography in the assessment of aortic valve morphology: pathological correlation in 178 patients. Can J Cardiol. 1999;15:48-52.

25. Brandenburg Jr RO, Tajik AJ, Edwards WD, et al. Accuracy of 2-dimensional echocardiographic diagnosis of congenitally bicuspid aortic valve: echocardiographic-anatomic correlation in 115 patients. Am J Cardiol. 1983;51:1469-73. 